\title{
An epidemic CC1-MRSA-IV clone yields false-negative test results in molecular MRSA identification assays: a note of caution, Austria, Germany, Ireland, 2020
}

Stefan Monecke ${ }^{1,2,3,4}$, Elisabeth König ${ }^{4,5}$, Megan R Earls ${ }^{4,6}$, Eva Leitner ${ }^{5}$, Elke Müller ${ }^{1,3}$, Gabriel E Wagner ${ }^{5}$, David M Poitz ${ }^{7}$, Lutz Jatzwauk $^{8}$, Teodora Vremera ${ }^{9}$, Olivia S Dorneanu ${ }^{9}$, Alexandra Simbeck ${ }^{10}$, Andreas Ambrosch ${ }^{11}$, Ines Zollner-Schwetz ${ }^{12}$, Robert Krause $^{12}$, Werner Ruppitsch ${ }^{13}$, Wulf Schneider-Brachert ${ }^{10}$, David C Coleman ${ }^{6}$, Ivo Steinmetz ${ }^{5}$, Ralf Ehricht ${ }^{1,3,14}$

1. Leibniz Institute of Photonic Technology (IPHT), Jena, Germany

2. Institute for Medical Microbiology and Hygiene, Medical Faculty ‘Carl Gustav Carus', Technische Universität Dresden, Dresden, Germany

3. InfectoGnostics Research Campus Jena, Jena, Germany

4. These authors contributed equally

5. Diagnostic and Research Institute for Hygiene, Microbiology and Environmental Medicine, Medical University of Graz, Graz, Austria

6. Microbiology Research Unit, Division of Oral Biosciences, Dublin Dental University Hospital, Trinity College, University of Dublin, Dublin, Ireland

7. Institute of Clinical Chemistry and Laboratory Medicine, University Hospital 'Carl Gustav Carus', Technische Universität Dresden, Dresden, Germany

8. Department of Hospital Infection Control, University Hospital ‘Carl Gustav Carus’, Technische Universität Dresden, Dresden, Germany

9. Microbiology Unit, Department of Preventive and Interdisciplinary Medicine, University of Medicine and Pharmacy 'Grigore T Popa', Iaşi, Romania

10. Department of Infection Control and Infectious Diseases, University Hospital Regensburg, Regensburg, Germany

11. Institute of Laboratory Medicine, Microbiology and Hygiene, Barmherzige Brüder Hospital, Regensburg, Germany

12. Section of Infectious Diseases and Tropical Medicine, Department of Internal Medicine, Medical University of Graz, Austria

13. Institute of Medical Microbiology and Hygiene, Austrian Agency for Health and Food Safety, Vienna, Austria

14. Friedrich- Schiller University; Institute of Physical Chemistry, Jena, Germany

Correspondence: Eva Leitner (eva.leitner@medunigraz.at)

Citation style for this article:

Monecke Stefan, König Elisabeth, Earls Megan R, Leitner Eva ORCID icon, Müller Elke, Wagner Gabriel E, Poitz David M , Jatzwauk Lutz, Vremera Teodora,

Dorneanu Olivia S, Simbeck Alexandra, Ambrosch Andreas, Zollner-Schwetz Ines, Krause Robert, Ruppitsch Werner, Schneider-Brachert Wulf, Coleman David C

, Steinmetz Ivo, Ehricht Ralf. An epidemic CC 1-MRSA-IV clone yields false-negative test results in molecular MRSA identification assays: a note of caution, Austria, Germany, Ireland, 2020. Euro Surveill. 2020;25(25):pii=2000929. https://doi.org/10.2807/1560-7917.ES.2020.25.25.2000929

\footnotetext{
Article submitted on 14 May 2020 / accepted on 18 Jun 2020 / published on 25 June 2020
}

We investigated why a clinical meticillin-resistant Staphylococcus aureus (MRSA) isolate yielded false-negative results with some commercial PCR tests for MRSA detection. We found that an epidemic European CC1-MRSA-IV clone generally exhibits this behaviour. The failure of the assays was attributable to a large insertion in the orfX/SCCmec integration site. To ensure the reliability of molecular MRSA tests, it is vital to monitor emergence of new SCCmec types and junction sites.

We investigated why a clinical meticillin-resistant Staphylococcus aureus (MRSA) isolate, collected in Austria in late 2019, yielded false-negative results with two widely used commercial orfX/SCCmec junction assays (Cepheid GeneXpert MRSA/SA BC, BD MAX Staph SR). The aim of this study was to investigate false-negative results with these two assays. Therefore, we tested and sequenced the index isolate and other isolates assigned by microarray to the same strain (i.e. the European CC1-MRSA-IV [1]).

\section{Index case}

A 62-year-old patient with metastasised cancer was admitted with suspected pneumonia to the Medical University Hospital of Graz, Austria. Blood cultures (BACTEC, Becton Dickinson, Heidelberg, Germany) became rapidly positive and Staphylococcus aureus was identified by in situ hybridisation (PNA FISH, AdvanDx, Woburn, United States (US)). In order to identify meticillin-resistant $S$. aureus (MRSA), the blood culture was investigated using the GeneXpert MRSA/SA BC PCR (Cepheid, Sunnyvale, US). Simultaneously, rapid antimicrobial susceptibility testing (RAST) was performed [2,3]. The GeneXpert test was negative for MRSA but RAST revealed cefoxitin resistance after 6 h. Antimicrobial susceptibility testing (Vitek2, bioMérieux, Marcy-l'Étoile, France) confirmed meticillin resistance. BD MAX StaphSR (Becton Dickinson) yielded a negative MRSA result. Microarray-based characterisation (S. aureus Genotyping Kit 2.0, Abbott, Alere Technologies, Jena, Germany) detected mecA and assigned the isolate (Graz_511421-19) to clonal complex CC1-MRSA-IV. Although the antibiotic 
TABLE 1A

MRSA strains and isolates investigated in the present study and their detection using commercially available orfX/SCCmec junction site assays $(\mathrm{n}=47)$

\begin{tabular}{|c|c|c|c|c|c|}
\hline Isolate & $\begin{array}{l}\text { Strain affiliation } \\
\text { (according to microarray) }\end{array}$ & $\mathrm{SCC} m e c$ element & Origin & $\mathrm{BD}$ MAX results & GeneXpert results \\
\hline $\begin{array}{l}\text { Graz_511421-19 } \\
\text { (index case) }\end{array}$ & $\begin{array}{l}\text { CC1-MRSA-IV (PVL-neg, } \\
\text { aphA3/sat-pos) }\end{array}$ & $\begin{array}{l}\mathrm{SCCmec} \text { IVa with } \\
\text { insertion }\end{array}$ & Austria, 2019 & Negative (G) & Negative $(2 \mathrm{X} \mathrm{BC} ; \mathrm{G})$ \\
\hline Dresden-94757 & $\begin{array}{c}\text { CC1-MRSA-IV (PVL-neg, } \\
\text { aphA3/sat-pos) }\end{array}$ & $\begin{array}{c}\mathrm{SCC} \text { sec IVa with } \\
\text { insertion }\end{array}$ & Saxony, 2010 & Negative (D, G) & $\mathrm{N} / \mathrm{A}$ \\
\hline Dresden-94758 & $\begin{array}{c}\text { CC1-MRSA-IV (PVL-neg, } \\
\text { aphA3/sat-pos) }\end{array}$ & $\begin{array}{c}\text { SCCmec IVa with } \\
\text { insertion }\end{array}$ & Saxony, 2014 & Negative (D, G) & $\mathrm{N} / \mathrm{A}$ \\
\hline Dresden-94759 & $\begin{array}{c}\text { CC1-MRSA-IV (PVL-neg, } \\
\text { aphA3/sat-pos) }\end{array}$ & $\begin{array}{c}\text { SCCmec IVa with } \\
\text { insertion }\end{array}$ & Saxony, 2009 & Negative (D, G) & $\mathrm{N} / \mathrm{A}$ \\
\hline Dresden-94760 & $\begin{array}{c}\text { CC1-MRSA-IV (PVL-neg, } \\
\text { aphA3/sat-pos) }\end{array}$ & $\begin{array}{c}\text { SCCmec IVa with } \\
\text { insertion }\end{array}$ & Saxony, 2010 & Negative (D, G) & $\mathrm{N} / \mathrm{A}$ \\
\hline lasi-95033 & $\begin{array}{c}\text { CC1-MRSA-IV (PVL-neg, } \\
\text { aphA3/sat-pos) }\end{array}$ & $\begin{array}{l}\text { SCCmec IVa with } \\
\text { insertion }\end{array}$ & Romania, 2009 & Negative (D, G) & $\mathrm{N} / \mathrm{A}$ \\
\hline Iasi-95034 & $\begin{array}{c}\text { CC1-MRSA-IV (PVL-neg, } \\
\text { aphA3/sat-pos) }\end{array}$ & $\begin{array}{c}\text { SCCmec IVa with } \\
\text { insertion }\end{array}$ & Romania, 2009 & $\begin{array}{c}\text { Negative }(\mathrm{D}) / \\
\text { ambiguous (G) }\end{array}$ & $\mathrm{N} / \mathrm{A}$ \\
\hline lasi-95035 & $\begin{array}{c}\text { CC1-MRSA-IV (PVL-neg, } \\
\text { aphA3/sat-pos) }\end{array}$ & $\begin{array}{c}\mathrm{SCC} \text { mec IVa with } \\
\text { insertion }\end{array}$ & Romania, 2009 & $\begin{array}{c}\text { Negative (D)/ } \\
\text { ambiguous (G) }\end{array}$ & $\mathrm{N} / \mathrm{A}$ \\
\hline Iasi-95037 & $\begin{array}{c}\text { CC1-MRSA-IV (PVL-neg, } \\
\text { aphA3/sat-pos) }\end{array}$ & $\begin{array}{c}\text { SCCmec IVa with } \\
\text { insertion }\end{array}$ & Romania, 2009 & Negative (D, G) & $\mathrm{N} / \mathrm{A}$ \\
\hline Iasi-95038 & $\begin{array}{c}\text { CC1-MRSA-IV (PVL-neg, } \\
\text { aphA3/sat-pos) }\end{array}$ & $\begin{array}{c}\text { SCCmec IVa with } \\
\text { insertion }\end{array}$ & Romania, 2009 & Negative (D, G) & $\mathrm{N} / \mathrm{A}$ \\
\hline lasi-95039 & $\begin{array}{c}\text { CC1-MRSA-IV (PVL-neg, } \\
\text { aphA3/sat-pos) }\end{array}$ & $\begin{array}{l}\mathrm{SCC} \text { mec IVa with } \\
\text { insertion }\end{array}$ & Romania, 2009 & $\begin{array}{c}\text { Negative (D)/ } \\
\text { ambiguous (G)a }\end{array}$ & $\mathrm{N} / \mathrm{A}$ \\
\hline Iasi-95040 & $\begin{array}{l}\text { CC1-MRSA-IV (PVL-neg, } \\
\text { aphA3/sat-pos) }\end{array}$ & $\begin{array}{l}\mathrm{SCC} \text { mec IVa with } \\
\text { insertion }\end{array}$ & Romania, 2009 & Negative (D, G) & $\mathrm{N} / \mathrm{A}$ \\
\hline lasi-95041 & $\begin{array}{c}\text { CC1-MRSA-IV (PVL-neg, } \\
\text { aphA3/sat-pos) }\end{array}$ & $\begin{array}{l}\text { SCCmec IVa with } \\
\text { insertion }\end{array}$ & Romania, 2009 & Negative (D, G) & $\mathrm{N} / \mathrm{A}$ \\
\hline Iasi-174752 & $\begin{array}{c}\text { CC1-MRSA-IV (PVL-neg, } \\
\text { aphA3/sat-pos) }\end{array}$ & $\begin{array}{c}\text { SCCmec IVa with } \\
\text { insertion }\end{array}$ & Romania, 2010 & Negative (D, G) & $\mathrm{N} / \mathrm{A}$ \\
\hline Iasi-176047 & $\begin{array}{c}\text { CC1-MRSA-IV (PVL-neg, } \\
\text { aphA3/sat-pos) }\end{array}$ & $\begin{array}{c}\text { SCCmec IVa with } \\
\text { insertion }\end{array}$ & Romania, 2009 & Negative (D, G) & $\mathrm{N} / \mathrm{A}$ \\
\hline Bavaria-0643 & $\begin{array}{c}\text { CC1-MRSA-IV (PVL-neg, } \\
\text { aphA3/sat-pos) }\end{array}$ & $\begin{array}{c}\text { SCCmec IVa with } \\
\text { insertion }\end{array}$ & Bavaria, 2018 & $\mathrm{~N} / \mathrm{A}$ & Positive (SSTI; R) \\
\hline Bavaria-0824 & $\begin{array}{c}\text { CC1-MRSA-IV (PVL-neg, } \\
\text { aphA3/sat-pos) }\end{array}$ & $\begin{array}{c}\text { SCCmec IVa with } \\
\text { insertion }\end{array}$ & Bavaria, 2015 & N/A & Positive (SSTI; R) \\
\hline Bavaria-1185 & $\begin{array}{c}\text { CC1-MRSA-IV (PVL-neg, } \\
\text { aphA3/sat-pos) }\end{array}$ & $\begin{array}{c}\text { SCCmec IVa with } \\
\text { insertion }\end{array}$ & Bavaria, 2018 & N/A & Positive (SSTI; R) \\
\hline Bavaria-1274 & $\begin{array}{c}\text { CC1-MRSA-IV (PVL-neg, } \\
\text { aphA3/sat-pos) }\end{array}$ & $\begin{array}{c}\text { SCCmec IVa with } \\
\text { insertion }\end{array}$ & Bavaria, 2014 & $\mathrm{~N} / \mathrm{A}$ & Positive (SSTI; R) \\
\hline Bavaria-1537 & $\begin{array}{c}\text { CC1-MRSA-IV (PVL-neg, } \\
\text { aphA3/sat-pos) }\end{array}$ & $\begin{array}{l}\mathrm{SCC} \text { sec IVa with } \\
\text { insertion }\end{array}$ & Bavaria, 2013 & $\mathrm{~N} / \mathrm{A}$ & Positive (SSTI; R) \\
\hline Bavaria-178o & $\begin{array}{c}\text { CC1-MRSA-IV (PVL-neg, } \\
\text { aphA3/sat-pos) }\end{array}$ & $\begin{array}{c}\mathrm{SCC} \text { mec IVa with } \\
\text { insertion }\end{array}$ & Bavaria, 2013 & $\mathrm{~N} / \mathrm{A}$ & Positive (SSTI; R) \\
\hline Bavaria-1962 & $\begin{array}{c}\text { CC1-MRSA-IV (PVL-neg, } \\
\text { aphA3/sat-pos) }\end{array}$ & $\begin{array}{c}\text { SCCmec IVa with } \\
\text { insertion }\end{array}$ & Bavaria & $\mathrm{N} / \mathrm{A}$ & Positive (SSTI; R) \\
\hline Bavaria-2102 & $\begin{array}{c}\text { CC1-MRSA-IV (PVL-neg, } \\
\text { aphA3/sat-pos) }\end{array}$ & $\begin{array}{c}\text { SCCmec IVa with } \\
\text { insertion }\end{array}$ & Bavaria, 2019 & $\mathrm{~N} / \mathrm{A}$ & Positive (SSTI; R) \\
\hline Bavaria-2220 & $\begin{array}{c}\text { CC1-MRSA-IV (PVL-neg, } \\
\text { aphA3/sat-pos) }\end{array}$ & $\begin{array}{c}\text { SCCmec IVa with } \\
\text { insertion }\end{array}$ & Bavaria & $\mathrm{N} / \mathrm{A}$ & Positive (SSTI; R) \\
\hline Bavaria-2312 & $\begin{array}{c}\text { CC1-MRSA-IV (PVL-neg, } \\
\text { aphA3/sat-pos) }\end{array}$ & $\begin{array}{c}\text { SCCmec IVa with } \\
\text { insertion }\end{array}$ & Bavaria & $\mathrm{N} / \mathrm{A}$ & Positive (SSTI; R) \\
\hline Bavaria-2360 & $\begin{array}{c}\text { CC1-MRSA-IV (PVL-neg, } \\
\text { aphA3/sat-pos) }\end{array}$ & $\begin{array}{c}\text { SCCmec IVa with } \\
\text { insertion }\end{array}$ & Bavaria & $\mathrm{N} / \mathrm{A}$ & Positive (SSTI; R) \\
\hline Bavaria-2391 & $\begin{array}{c}\text { CC1-MRSA-IV (PVL-neg, } \\
\text { aphA3/sat-pos) }\end{array}$ & $\begin{array}{c}\mathrm{SCC} \text { mec IVa with } \\
\text { insertion }\end{array}$ & Bavaria, 2018 & $\mathrm{~N} / \mathrm{A}$ & Positive (SSTI; R) \\
\hline Bavaria-2483 & $\begin{array}{c}\text { CC1-MRSA-IV (PVL-neg, } \\
\text { aphA3/sat-pos) }\end{array}$ & $\begin{array}{c}\text { SCCmec IVa with } \\
\text { insertion }\end{array}$ & Bavaria, 2019 & $\mathrm{~N} / \mathrm{A}$ & Positive (SSTI; R) \\
\hline Bavaria-2535 & $\begin{array}{c}\text { CC1-MRSA-IV (PVL-neg, } \\
\text { aphA3/sat-pos) }\end{array}$ & $\begin{array}{c}\text { SCCmec IVa with } \\
\text { insertion }\end{array}$ & Bavaria, 2019 & $\mathrm{~N} / \mathrm{A}$ & Positive (SSTI; R) \\
\hline Bavaria-2584 & $\begin{array}{c}\text { CC1-MRSA-IV (PVL-neg, } \\
\text { aphA3/sat-pos) }\end{array}$ & $\begin{array}{l}\text { SCCmec IVa with } \\
\text { insertion }\end{array}$ & Bavaria, 2019 & $\mathrm{~N} / \mathrm{A}$ & Positive (SSTI; R) \\
\hline
\end{tabular}


MRSA strains and isolates investigated in the present study and their detection using commercially available orfX/SCCmec junction site assays $(\mathrm{n}=47)$

\begin{tabular}{|c|c|c|c|c|c|}
\hline Isolate & $\begin{array}{c}\text { Strain affiliation } \\
\text { (according to microarray) }\end{array}$ & $\mathrm{SCC} m e c$ element & Origin & BD MAX results & GeneXpert results \\
\hline Bavaria-2585 & $\begin{array}{l}\text { CC1-MRSA-IV (PVL-neg, } \\
\text { aphA3/sat-pos) }\end{array}$ & $\begin{array}{l}\mathrm{SCC} m e c \text { IVa with } \\
\text { insertion }\end{array}$ & Bavaria, 2019 & $\mathrm{~N} / \mathrm{A}$ & Positive (SSTI; R) \\
\hline Bavaria-2588 & $\begin{array}{c}\text { CC1-MRSA-IV (PVL-neg, } \\
\text { aphA3/sat-pos) }\end{array}$ & $\begin{array}{l}\text { SCCmec IVa with } \\
\text { insertion }\end{array}$ & Bavaria, 2019 & $\mathrm{~N} / \mathrm{A}$ & Positive (SSTI; R) \\
\hline Bavaria-2596 & $\begin{array}{c}\text { CC1-MRSA-IV (PVL-neg, } \\
\text { aphA3/sat-pos) }\end{array}$ & $\begin{array}{l}\text { SCCmec IVa with } \\
\text { insertion }\end{array}$ & Bavaria, 2019 & $\mathrm{~N} / \mathrm{A}$ & Positive (SSTI; R) \\
\hline Bavaria-2618 & $\begin{array}{c}\text { CC1-MRSA-IV (PVL-neg, } \\
\text { aphA3/sat-pos) }\end{array}$ & $\begin{array}{l}\text { SCCmec IVa with } \\
\text { insertion }\end{array}$ & Bavaria, 2012 & $\mathrm{~N} / \mathrm{A}$ & Positive (SSTI; R) \\
\hline Bavaria-3012 & $\begin{array}{c}\text { CC1-MRSA-IV (PVL-neg, } \\
\text { aphA3/sat-pos) }\end{array}$ & $\begin{array}{l}\text { SCCmec IVa with } \\
\text { insertion }\end{array}$ & Bavaria, 2011 & $\mathrm{~N} / \mathrm{A}$ & Positive (SSTI; R) \\
\hline Bavaria-3254 & $\begin{array}{c}\text { CC1-MRSA-IV (PVL-neg, } \\
\text { aphA3/sat-pos) }\end{array}$ & $\begin{array}{l}\text { SCCmec IVa with } \\
\text { insertion }\end{array}$ & Bavaria, 2010 & $\mathrm{~N} / \mathrm{A}$ & Positive (SSTI; R) \\
\hline Bavaria-3702 & $\begin{array}{c}\text { CC1-MRSA-IV (PVL-neg, } \\
\text { aphA3/sat-pos) }\end{array}$ & $\begin{array}{l}\text { SCCmec IVa with } \\
\text { insertion }\end{array}$ & Bavaria, 2019 & $\mathrm{~N} / \mathrm{A}$ & Positive (SSTI; R) \\
\hline Bavaria-3741 & $\begin{array}{c}\text { CC1-MRSA-IV (PVL-neg, } \\
\text { aphA3/sat-pos) }\end{array}$ & $\begin{array}{l}\mathrm{SCC} m e c \text { IVa with } \\
\text { insertion }\end{array}$ & Bavaria, 2019 & $\mathrm{~N} / \mathrm{A}$ & Positive (SSTI; R) \\
\hline Bavaria-3784 & $\begin{array}{c}\text { CC1-MRSA-IV (PVL-neg, } \\
\text { aphA3/sat-pos) }\end{array}$ & $\begin{array}{c}\mathrm{SCC} m e c \text { IVa with } \\
\text { insertion }\end{array}$ & Bavaria, 2019 & $\mathrm{~N} / \mathrm{A}$ & Positive (SSTI; R) \\
\hline Dresden-220663 & $\begin{array}{c}\text { CC1-MRSA-IV, (PVL-neg, } \\
\text { aphA3/sat-neg) }\end{array}$ & $\begin{array}{l}\mathrm{SCC} \text { mec IVa (as in } \\
\text { reference strain } \mathrm{MW} \text { 2) }\end{array}$ & Saxony, 2007 & Positive (D) & N/A \\
\hline Dresden-124288 & $\begin{array}{c}\text { CC22-MRSA-IV (Barnim/ } \\
\text { UK EMRSA-15) }\end{array}$ & $\mathrm{ScCmec} \mathrm{IVh} / \mathrm{j}$ & Saxony & Positive (D) & $\mathrm{N} / \mathrm{A}$ \\
\hline Dresden-124289 & $\begin{array}{c}\text { CC22-MRSA-IV (Barnim/ } \\
\text { UK EMRSA-15) }\end{array}$ & $\mathrm{Sccmec} \mathrm{IVh} / \mathrm{j}$ & Saxony & Positive (D) & $\mathrm{N} / \mathrm{A}$ \\
\hline Dresden-124281 & $\begin{array}{c}\text { CC45-MRSA-IV (Berlin } \\
\text { EMRSA) }\end{array}$ & SCCmec IVa & Saxony & Positive (D) & $\mathrm{N} / \mathrm{A}$ \\
\hline $\mathrm{MU}_{50}$ & $\begin{array}{c}\text { CC5-MRSA-II (New York/ } \\
\text { Japan clone) }\end{array}$ & $\mathrm{ScCmec} \mathrm{II}$ & \begin{tabular}{|l|}
$\begin{array}{c}\text { Japan (sequenced } \\
\text { reference strain) }\end{array}$ \\
\end{tabular} & Positive (D) & $N / A$ \\
\hline$M W_{2}$ & $\begin{array}{c}\text { CC1-MRSA-IV (PVL-pos } \\
\text { US400) }\end{array}$ & SCCmec IVa & $\begin{array}{c}\text { United States } \\
\text { (sequenced } \\
\text { reference strain) }\end{array}$ & Positive (D) & $\mathrm{N} / \mathrm{A}$ \\
\hline N315 & $\begin{array}{c}\text { CC5-MRSA-II (New York/ } \\
\text { Japan clone) }\end{array}$ & $\mathrm{ScCmec} \mathrm{II}$ & $\begin{array}{c}\text { Japan (sequenced } \\
\text { reference strain) }\end{array}$ & Positive (D) & $\mathrm{N} / \mathrm{A}$ \\
\hline US300-FPR3757 & $\begin{array}{c}\text { CC8-MRSA-[Iva- } \\
\text { posACME1] (PVL-pos), } \\
\text { US300 }\end{array}$ & $\begin{array}{c}\text { SCC [mec } \\
\text { IVa+ACME1+Cu] }\end{array}$ & $\begin{array}{l}\text { US (GenBank } \\
\text { CPoo0255.1) }\end{array}$ & Positive (D) & $\mathrm{N} / \mathrm{A}$ \\
\hline
\end{tabular}

BC: GeneXpert MRSA/SA BC; CC: clonal complex; MRSA: meticillin-resistant Staphylococcus aureus; EMRSA: epidemic strain of MRSA; N/A: not available; PVL: Panton-Valentine leukocidin; SCCmec: staphylococcal cassette chromosome mec; SSTI: GeneXpert MRSA/SA SSTI.

$D, G$, and R indicate that assays were performed in Dresden, Graz and Regensburg, respectively.

${ }^{a}$ Ambiguous: weak signal observed at cycle threshold $\mathrm{C} t>35$.

treatment was adapted, the patient died shortly after because of tumour progression.

\section{Isolates}

Ten CC1-MRSA-IV isolates originated from the Sfanta Parascheva Hospital, lasi, in north-eastern Romania [4]. Four isolates originated from the Dresden University Hospital, Saxony, Germany. Nineteen isolates from Regensburg University Medical Centre in Bavaria, Germany and five isolates from other Bavarian hospitals were also included. All included isolates had been collected and preliminarily analysed as part of earlier collaborations. Four fully sequenced reference strains were used as controls (Table 1). Additional controls comprised four isolates from Dresden that belonged to local epidemic strains or to another $\mathrm{CC}_{1}$-MRSA strain (isolate Dresden-220663).

\section{Commercial MRSA assays}

Test results are provided in Table 1. The index isolate tested negative using the BD MAX Staph SR assay (Lot 9303156). Isolates from lasi and Dresden and controls were tested twice, in Graz and Dresden, using this assay (Graz, Lot 9303156; Dresden, Lot K55928980720210312). It failed to identify these 15 isolates although controls handled in parallel were correctly identified.

Testing of the index isolate with GeneXpert MRSA/SA BC (Lots 1000148707 and 1000179462) yielded negative results, too. Further investigations on this assay were not possible because that laboratory became involved in diagnostics for the coronavirus (Covid-19) pandemic. 
TABLE 2A

Genes in the variant SCCmec IVa element of the European CC1-MRSA-IV strain

\begin{tabular}{|c|c|c|c|c|c|}
\hline Gene ID & $\begin{array}{l}\text { Definition of gene product and } \\
\text { comments (see also annotation of } \\
\text { MH188467.1) }\end{array}$ & Orientation & $\begin{array}{l}\text { Locus tag in MW2 } \\
\text { (BA000033.2) }\end{array}$ & $\begin{array}{l}\text { Nucleotide positions } \\
\text { in GenBank RBVO }\end{array}$ & $\begin{array}{c}\text { Nucleotide positions } \\
\text { in GenBank MT380478 } \\
\text { (Iasi-95037) }\end{array}$ \\
\hline orfX & $23 \mathrm{~S}$ rRNA methyltransferase & Forward & MWoo24 & $\begin{array}{l}\text { RBVO01000005.1; nt } \\
280,209-280,689\end{array}$ & $\mathrm{~N} / \mathrm{A}$ \\
\hline sRNA6 & Antisense RNA associated with orfX & Reverse & $\mathrm{N} / \mathrm{A}$ & $\begin{array}{l}\text { RBVO01000005.1; nt } \\
280,389-280,673\end{array}$ & $\mathrm{~N} / \mathrm{A}$ \\
\hline DR_SCC & $\begin{array}{c}\text { Direct repeat of SCC, to } 19 \mathrm{nt} \text { of the } 3^{6} \\
\text { end of the coding sequence of orfX }\end{array}$ & $\mathrm{N} / \mathrm{A}$ & $\mathrm{N} / \mathrm{A}$ & $\begin{array}{l}\text { RBVO01000005.1; nt } \\
280,670-280,689\end{array}$ & nt $1-19$ \\
\hline sccterm15 & $\begin{array}{l}\text { SCC-terminal sequence adjacent } \\
\text { to orfX, and alternate to dcs, see } \\
\text { Discussion }\end{array}$ & $\mathrm{N} / \mathrm{A}$ & Not present & $\begin{array}{l}\text { RBVO01000005.1; nt } \\
280,689-280,912\end{array}$ & nt $20-242$ \\
\hline $\mathrm{E}_{7} \mathrm{MHX}_{1}$ & Transcription regulator & Forward & Not present & $\begin{array}{l}\text { RBVO01000005.1; nt } \\
280,912-281,239\end{array}$ & nt $243-569$ \\
\hline ydil2 & $\begin{array}{c}\text { Hypothetical protein/putative } \\
\text { membrane peptidase, associated } \\
\text { with SCC elements }\end{array}$ & Forward & Not present & $\begin{array}{l}\text { RBVO01000005.1; nt } \\
281,275-282,109\end{array}$ & nt $606-1,439$ \\
\hline C5QAP8-M299 & Hypothetical protein & Forward & Not present & $\begin{array}{l}\text { RBVO01000005.1; nt } \\
282,794-283,568\end{array}$ & nt $2,125-2,898$ \\
\hline $\mathrm{A}_{8 Y Y X_{4}}$ & Hypothetical protein & Reverse & Not present & $\begin{array}{l}\text { RBVO01000005.1; nt } \\
283,805-284,144\end{array}$ & nt $3,136-3,474$ \\
\hline$n p d-\mathrm{SCC}$ & $\begin{array}{l}\text { Enoyl-[acyl-carrier-protein] } \\
\text { reductase-like protein }\end{array}$ & Reverse & Not present & $\begin{array}{l}\text { RBVO01000005.1; nt } \\
284,329-285,400\end{array}$ & nt $3,660-4,730$ \\
\hline $\mathrm{H}_{4} \mathrm{AYD} 7$-trunc & Transcriptional regulator, LysR family & Truncated & Not present & $\begin{array}{l}\text { RBVO01000005.1; nt } \\
285,412-286,024\end{array}$ & nt $4,743-5,354$ \\
\hline Q7A213-trunc & $\begin{array}{l}\text { Putative protein; it comprises the } \\
\text { inverted repeat of IS } 431 . \text { In MW2 it is } \\
\text { not truncated and comprises } 240 \mathrm{nt}\end{array}$ & Truncated & MWoo26 & $\begin{array}{l}\text { RBVO01000005.1; nt } \\
286,024-286,052\end{array}$ & nt $5,355-5,382$ \\
\hline IR_IS431 & Inverted repeat of IS431 & Truncated & $\mathrm{N} / \mathrm{A}$ & $\begin{array}{l}\text { RBVO01000005.1; nt } \\
286,024-286,040\end{array}$ & nt $5,355-5,370$ \\
\hline tnplS431 & Transposase for IS 431 & Reverse & MWoo27 & $\begin{array}{l}\text { RBV001000005.1; nt } \\
286,083 \text {-end of contig } \\
\text { (nt 286,184) (partial) }\end{array}$ & nt $5,414-6,088$ \\
\hline Teg143 & $\begin{array}{l}\text { Trans-encoded RNA associated with } \\
\text { tnplS } 431\end{array}$ & Forward & $\mathrm{N} / \mathrm{A}$ & $\begin{array}{l}\text { RBVO01000003.1; nt } \\
203-237\end{array}$ & nt $6,119-6,152$ \\
\hline IR_IS431 & Inverted repeat of IS 431 & Truncated & $\mathrm{N} / \mathrm{A}$ & $\begin{array}{c}\text { RBV001000003.1; nt } \\
213-229\end{array}$ & nt $6,129-6,144$ \\
\hline mvas-scC & Truncated HMG-CoA synthase & Forward & MWoo28 & $\begin{array}{c}\text { RBVO01000003.1; nt } \\
245-598 \\
\end{array}$ & nt $6,161-6,513$ \\
\hline Q5HJW6 & Hypothetical protein & Forward & $\mathrm{N} / \mathrm{A}$ & $\begin{array}{l}\text { RBVO01000003.1; nt } \\
695-1,046\end{array}$ & nt $6,611-6,841$ \\
\hline$d r u$ & SCC direct repeat units & Truncated & $\mathrm{N} / \mathrm{A}$ & $\begin{array}{l}\text { RBVO01000003.1; nt } \\
835-1,273\end{array}$ & nt $6,751-7,148$ \\
\hline ugpQ & $\begin{array}{c}\text { Glycerophosphoryl diester } \\
\text { phosphodiesterase-like protein }\end{array}$ & Forward & MWoo29 & $\begin{array}{c}\text { RBVO01000003.1; nt } \\
1,474-2,218\end{array}$ & nt $7,350-8,093$ \\
\hline ydeM & Acyl dehydratase $\mathrm{MaoC}$ & Forward & MWo030 & $\begin{array}{c}\text { RBVO01000003.1; nt } \\
2,314-2,743\end{array}$ & nt $8,190-8,618$ \\
\hline mecA & $\begin{array}{c}\text { Encodes penicillin binding protein } 2 \\
\text { prime, defining MRSA }\end{array}$ & Reverse & MWo031 & $\begin{array}{c}\text { RBVO01000003.1; nt } \\
2,812-4,795\end{array}$ & nt $8,664-10,670$ \\
\hline mecR1-trunc & $\begin{array}{l}\text { Meticillin resistance operon repressor } \\
\text { 1, signal transducer protein, } \\
\text { truncated in SCCmec IV }\end{array}$ & Truncated & MWo032 & $\begin{array}{l}\text { RBVO01000003.1; nt } \\
4,894-5,862\end{array}$ & nt $10,770-10,816$ \\
\hline hsdR2-IS 1272 & \begin{tabular}{|c|} 
Type I site-specific deoxyribonuclease \\
restriction subunit
\end{tabular} & Truncated & MWo033 & $\begin{array}{c}\text { RBVO01000003.1; nt } \\
5,869-6,103\end{array}$ & nt $11,745-11,978$ \\
\hline tnpIS1272 & Transposase & Reverse & MWo034 & $\begin{array}{c}\text { RBVO01000003.1; nt } \\
6,103-7,627\end{array}$ & nt $11,979-13,502$ \\
\hline $\mathrm{Q}_{9} \mathrm{KX}_{75}$ & Hypothetical protein & Reverse & MWo035 & $\begin{array}{l}\text { RBV001000003.1; nt } \\
7,762-8,269\end{array}$ & nt $13,638-14,144$ \\
\hline $\mathrm{Q}_{7} \mathrm{~A} 207$ & Hypothetical protein & Reverse & MWo036 & $\begin{array}{l}\text { RBV001000003.1; nt } \\
8,283-8,595\end{array}$ & nt $14,159-14,470$ \\
\hline Q7A206-trunc & Hypothetical protein, truncated & Truncated & $\mathrm{N} / \mathrm{A}$ & $\begin{array}{l}\text { RBVO01000003.1; nt } \\
8,596-8,683\end{array}$ & nt $14,472-14,558$ \\
\hline Q7A206 & Hypothetical protein & Reverse & MWoo37 & $\begin{array}{l}\text { RBV001000003.1; nt } \\
8,681-9,032\end{array}$ & nt $14,557-14,907$ \\
\hline
\end{tabular}


Genes in the variant SCCmec IVa element of the European CC1-MRSA-IV strain

\begin{tabular}{|c|c|c|c|c|c|}
\hline Gene ID & $\begin{array}{c}\text { Definition of gene product and } \\
\text { comments (see also annotation of } \\
\text { MH188467.1) }\end{array}$ & Orientation & $\begin{array}{l}\text { Locus tag in } \mathrm{MW} 2 \\
\text { (BA000033.2) }\end{array}$ & $\begin{array}{l}\text { Nucleotide positions } \\
\text { in GenBank RBVO }\end{array}$ & $\begin{array}{l}\text { Nucleotide positions } \\
\text { in GenBank MT380478 } \\
\text { (Iasi-95037) }\end{array}$ \\
\hline UTR_ccrB-2 & $\begin{array}{l}\text { Highly conserved } 3^{6} \text {-untranslated } \\
\text { region of } c c r B\end{array}$ & $\mathrm{~N} / \mathrm{A}$ & $\mathrm{N} / \mathrm{A}$ & $\begin{array}{l}\text { RBVO01000003.1; nt } \\
9,032-9,553 \\
\end{array}$ & nt $14,908-15,428$ \\
\hline $\operatorname{ccrB}-2$ & $\begin{array}{c}\text { Cassette chromosome recombinase } \\
\text { B2 }\end{array}$ & Reverse & MWoo38 & $\begin{array}{l}\text { RBVO01000003.1; nt } \\
9,553-11,182\end{array}$ & nt $15,429-17,057$ \\
\hline CCrA-2 & $\begin{array}{l}\text { Cassette chromosome recombinase } \\
\mathrm{A}_{2}\end{array}$ & Reverse & MWoo39 & $\begin{array}{c}\text { RBVO01000003.1; nt } \\
11,203-12,553\end{array}$ & nt $17,079-18,428$ \\
\hline cch-2 & $\begin{array}{l}\text { Hypothetical protein/cassette } \\
\text { chromosome helicase }\end{array}$ & Reverse & MWoo40 & $\begin{array}{c}\text { RBVO01000003.1; nt } \\
12,786-14,574\end{array}$ & nt $18,662-20,449$ \\
\hline DUF1413 & $\begin{array}{c}\text { Hypothetical protein, associated with } \\
\text { cch }\end{array}$ & Reverse & MWoo41 & $\begin{array}{c}\text { RBVO01000003.1; nt } \\
14,573-14,864 \\
\end{array}$ & nt $20,449-20,739$ \\
\hline Q2FKL7 & Putative membrane protein & Forward & MWoo42 & $\begin{array}{c}\text { RBVO01000003.1; nt } \\
15,002-16,052\end{array}$ & nt $20,878-21,927$ \\
\hline Q8VUV8 & Putative transcriptional regulator & Forward & MWoo43 & $\begin{array}{c}\text { RBVO01000003.1; nt } \\
16,504-17,995\end{array}$ & nt $22,380-23,870$ \\
\hline CstB-SCC2 & $\begin{array}{c}\text { Includes a putative beta-lactamase; } \\
\text { marker for SCCmec IVa }\end{array}$ & Truncated & MWoo45 & $\begin{array}{c}\text { RBVO01000003.1; nt } \\
18,367-19,685\end{array}$ & nt $24,243-25,560$ \\
\hline $\mathrm{Q}_{2} \mathrm{FKL}_{3}$ & HNH endonuclease family protein & Forward & MWoo46 & $\begin{array}{c}\text { RBVO01000003.1; nt } \\
19,875-20,247\end{array}$ & nt $25,751-26,122$ \\
\hline Q8VUWo & Putative membrane protein & Forward & MWoo47 & $\begin{array}{l}\text { RBVO01000003.1; nt } \\
20,375-20,996\end{array}$ & nt $26,251-26,871$ \\
\hline DR_SCC & Direct repeat of SCC & Truncated & $\mathrm{N} / \mathrm{A}$ & $\begin{array}{c}\text { RBVO01000003.1; nt } \\
21,300-21,319\end{array}$ & nt $27,176-27,194$ \\
\hline
\end{tabular}

MRSA: meticillin-resistant Staphylococcus aureus; N/A: not available; nt: nucleotide position; SCC: staphylococcal cassette chromosome.

All Bavarian isolates were tested using Cepheid GeneXpert MRSA/SA SSTI (Lot 1000180532) that gave correct, positive results.

\section{Genotyping by microarray and sequencing} All isolates were genotyped using the $S$. aureus Genotyping Kit 2.0, a microarray covering 333 different target sequences corresponding to ca 170 different genes. Target genes, assay protocols and sequences of probes and primers have been published previously [5]. Isolates were assigned to clonal complexes, strains and SCCmec types based on microarray data as described [5].

All isolates underwent whole-genome sequencing. DNA was extracted as for array experiments. Its quality was assessed as previously described [6]. The Nextera DNA Flex Library Preparation Kit (Illumina, Eindhoven, the Netherlands) was used and libraries underwent pairedend sequencing using the 500-cycle MiSeq Reagent Kit v2 (Illumina). Libraries were scaled to exhibit at least 50-fold coverage. Sequencing run quality was assured following cluster density and Q30 assessment. Raw sequence reads were trimmed using fastp 0.19.11 [7] and assembled using SPAdes v3.9.1 [8]. Contigs under 1,000 bp were removed.

The sequence of the SCCmec element one representative isolate, lasi-95037, was deposited in GenBank (accession number: MT380478).

\section{Description of the strain and its SCCmec element}

Microarray profiling and genome sequencing showed that the index isolate belonged to a CC1-MRSA-IV clone previously described as 'European CC1-MRSA-IV' that may have emerged in south-eastern Europe $[1,4,9]$. A putative, meticillin-susceptible ancestor is common in Romania where this MRSA clone frequently observed already several years ago $[1,4]$. A high prevalence or outbreaks have been reported from Ireland [1], Italy [10] and Germany (North Rhine-Westphalia and Bavaria) $[1,11]$. In Regensburg, retrospective microarray-based typing of 3,067 isolates revealed that the occurrence of the European CC1-MRSA-IV clone increased from $<1 \%$ of typed MRSA between 2010 and 2013 to $9.4 \%$ in 2019. In Dresden, this strain has only sporadically been observed, accounting for seven in 1,758 isolates genotyped since 2000 ([1,12] and data not shown). Microarray genotyping data indicated that this clone was also recovered from horses and wild birds in Austria [13,14] and from livestock in Italy [15].

Isolates of this clone typically exhibit sequence type (ST)1 (1-1-1-1-1-1-1) or ST4110 (1-1-1-1-1-1-558) and spa types t127 (07-23-21-16-34-33-13), t386 (0723-13) or t1379o (07-23-21-16-34-33-34-34-33-34). Isolates usually carry ermC (erythromycin/clindamycin resistance), tetK (tetracycline resistance), aphA3 (kana-/ neomycin resistance), aadE (streptomycin resistance) and sat (streptothricin resistance). Some isolates harbour aacA-aphD (gentamicin resistance). Isolates from Ireland frequently exhibit resistance to mupirocin, 
SCCmec elements in the CC1 reference sequence MW2, the European CC1-MRSA-IV isolate Iasi-95037and the Staphylococcus epidermidis isolate P11PPP12

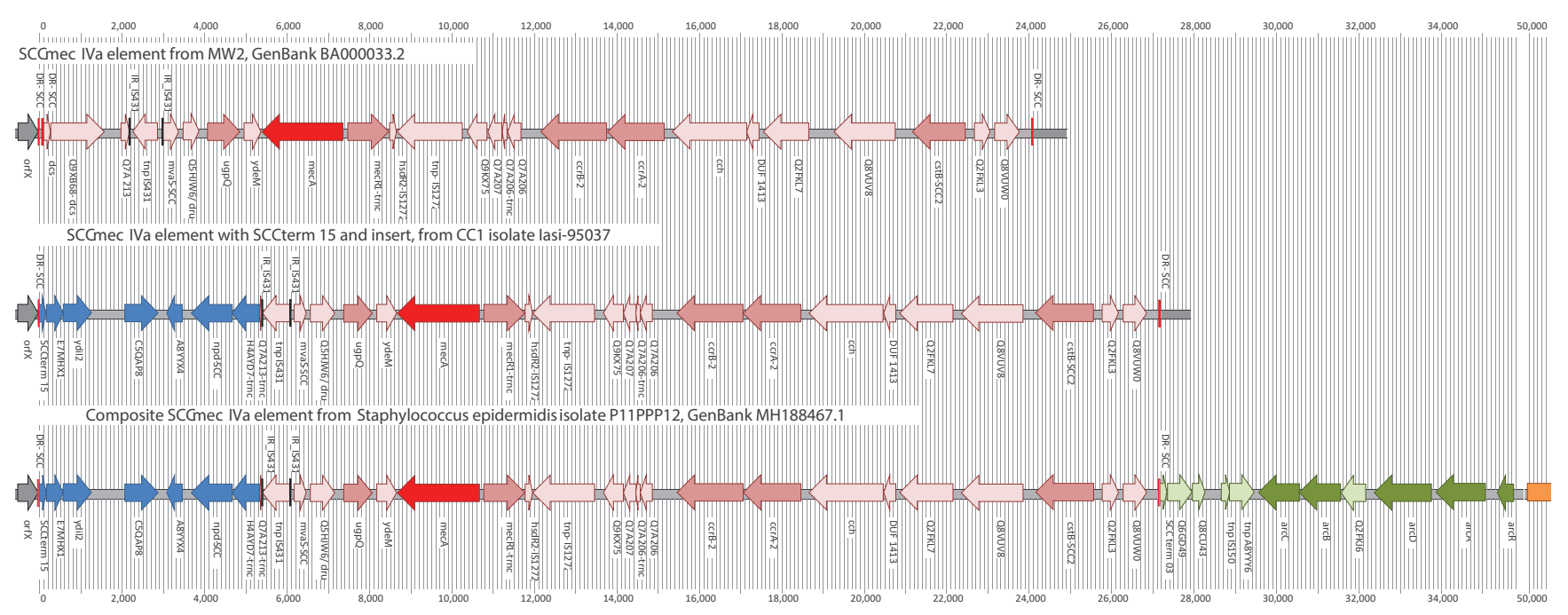

GenBank accession numbers: MW2: BAooo033.23; lasi-95037: MT380478; P11PPP12: MH188467.1.

chlorhexidine and quaternary ammonium compounds because of plasmid-borne iles2/mupR and qacA [1]. Fusidic acid resistance has not yet been detected in this clone, in contrast to other C 1 -MRSA of from the Middle East or the southern hemisphere.

The clone is PVL-negative and lacks the splE protease gene. It only rarely carries enterotoxin genes sek/ seq in addition to seh that is ubiquitously present in $\mathrm{CC}_{1}$. Its relationship to other $\mathrm{CC}_{1}$-MRSA clones has been discussed previously in detail [1].

This CC1-MRSA clone has an SCCmec IVa element which is essentially identical in all isolates and in a previous Irish sequence (GenBank RBVOoooooooo.1) [1]. In contrast to MW2 (BAooo033.2), it harbours an insertion of ca 5,350 nt, adjacent to orfX (Table 2, Figure). The insertion affects the orfX/SCCmec junction that is targeted by molecular tests for the detection of MRSA. It starts with a SCC terminal sequence alternate to $d c s$ ('SCCterm 15') and encodes six hypothetical proteins (E7MHX1, ydiL2, C5QAP8, A8YYX4, npd and H4AYD7; RBVOoooo05.1: 280,690-286,024). This insertion replaces $d c s / Q_{9} \mathrm{XB} 68-d c s$ and removes most (212 of $240 \mathrm{nt}$ ) of a gene encoding hypothetical protein Q7A213.

SCCterm 15 is present in at least three other MRSA strains. In CC152-MRSA-XIII, it is close to orfX (MG674089, CP024998), possibly affecting MRSA PCRs. In two other strains, it is situated within complex SCCmec elements [16]. In a Danish CC8MRSA strain (HMo30720.1), the same insert as in the European CC1-MRSA is localised between an ACME-II and an SCCmec IVa element [17,18]. A Saudi Arabian CC22-MRSA strain (HF569105.1) harbours SCCterm 15, E7MHX1, ydiL2, IR_IS431 and tnpIS431, localised between a copper resistance element and a composite ACME-II/ SCCmec IVh/j element [19].

An identical 5,350 nt cluster is present in Staphylococcus epidermidis $\mathrm{P}_{11} \mathrm{PPP}_{12}\left(\mathrm{MH}_{1}\right.$ 88467.1). Beyond that, the entire SCCmec IV element in $\mathrm{P}_{11} \mathrm{PPP}_{12}$ is identical to the one in the CC1-MRSA strain. Significantly, the site of recombination cutting short $\mathrm{Q}_{7} \mathrm{~A} 213$ is conserved in both strains (position 5,818/5,819 in the Supplement). Thus, it is likely that the entire SCCmec IVa cassette including the insert was transferred between ancestors of the two strains, i.e. across the species. However, $\mathrm{P}_{11} \mathrm{PPP}_{12}$ also harbours an ACME-II/heavy metal resistance element downstream of SCCmec, which is absent from the European C 1 -MRSA-IV. Therefore, it must have been lost during or after transfer of the SCCmec element, or it was acquired later by S. epidermidis.

\section{Discussion}

The study demonstrates that a CC1-MRSA-IV epidemic strain in Europe can yield false-negative results with common MRSA assays (GenXpert MRSA/SA BC, BD MAX Staph SR). Interestingly, GeneXpert MRSA/SA SSTI yielded correct results, indicating that the different tests utilise different primers. The absence of $d c s$ and coverage of SCCterm 15 appear to be the reason for the discrepancy.

False-negative results of PCRs targeting the orfX/ $\mathrm{SCC}$ mec junction site are concerning. Molecular assays are used to predict MRSA in positive blood cultures and to change therapy accordingly. The use of molecular assays is beneficial for a vast majority of patients because a result is available quickly. However, these assays can only detect target sequences that were available and considered at the time the primers were designed, and false-negative results have the potential 
to harm the patient by delaying effective therapy. Conventional antibiotic susceptibility tests are slower but are not constrained by the choice of primers or by the presence of unknown genotypes.

Molecular assays are also used to guide infection control. False-negative tests may result in lapses facilitating further MRSA transmission. Another, less obvious consequence might be a shift in the clonal structure of MRSA populations. When molecular assays exert a selective pressure favouring a false-negative strain, PCR-positive strains might get 'penalised' with subsequent interventions, hindering proliferation and transmission. This could lead to an increasing prevalence of the false-negative strain and to more failures in therapy and infection control.

It is crucial to monitor the emergence of new $\mathrm{SCC}$ mec junction sites in $S$. aureus and in coagulasenegative staphylococci, as mobile SCCmec elements can readily be transmitted between different strains and species, as was the case in the strain described here. Such unknown genotypes represent a problem for established molecular assays. As illustrated here, updating existing tests and platforms to evolving genotypes of the target organisms is important for individual and public health.

The containment of the $\mathrm{CC}_{1}$ strain must rely on conventional susceptibility tests, culture-based screening using selective growth media or updated molecular tests. We propose screening of medical or nursing staff recruited from epidemic regions, not only in hospitals but also in other care facilities, as well as patients with travel histories to these regions.

\section{Acknowledgments}

DC and ME wish to acknowledge the support of the staff of the Irish National MRSA Reference Laboratory at St. James's Hospital, Dublin, Ireland.

\section{Conflict of interest}

None declared.

\section{Authors' contributions}

Stefan Monecke: Conception and design of the study, data visualisation, analysis and interpretation of data, drafting of the manuscript. Elisabeth König: Collection of strains and data generation, analysis and interpretation of data. Megan R. Earls: Data generation, analysis and interpretation of data; drafting of the manuscript. Eva Leitner: Conception and design of the study, data generation, analysis and interpretation of data, drafting of the manuscript. Elke Müller: Data generation, analysis and interpretation of data. Gabriel Wagner: Data generation, analysis and interpretation of data. David Poitz: Data generation, analysis and interpretation of data. Lutz Jatzwauk: Data generation, analysis and interpretation of data. Teodora Vremera: Collection of strains and data generation. Olivia S. Dorneanu: Collection of strains and data generation. Alexandra Simbeck: Collection of strains and data generation, analysis and interpretation of data. Andreas Ambrosch: Data generation, analysis and interpretation of data. Ines Zollner-Schwetz: Data generation, analysis and interpretation of data. Robert Krause: Data generation, analysis and interpretation of data

Werner Ruppitsch: Data generation, analysis and interpretation of data. Wulf Schneider: Data generation, analysis and interpretation of data. David C. Coleman: Analysis and interpretation of data; drafting of the manuscript. Ivo Steinmetz: Analysis and interpretation of data; drafting of the manuscript. Ralf Ehricht: Conception and design of the study, analysis and interpretation of data; drafting of the manuscript,

\section{References}

1. Earls MR, Shore AC, Brennan GI, Simbeck A, SchneiderBrachert W, Vremera T, et al. A novel multidrug-resistant PVLnegative C 1 -MRSA-IV clone emerging in Ireland and Germany likely originated in South-Eastern Europe. Infect Genet Evol. 2019;69:117-26. https://doi.org/10.1016/j.meegid.2019.01.021 PMID: 30677533

2. The European Committee on Antimicrobial Susceptibility Testing (EUCAST). EUCAST rapid antimicrobial susceptibility testing (RAST) directly from positive blood culture bottles. Version 1.1. Växjö: EUCAST; 2019. Available from: https://www. eucast.org/fileadmin/src/media/PDFs/EUCAST_files/RAST/ EUCAST_RAST_methodology_v1.1_Final.pdf

3. The European Committee on Antimicrobial Susceptibility Testing (EUCAST). Zone diameter breakpoints for rapid antimicrobial susceptibility testing (RAST) directly from blood culture bottles. Version 1.1. Växjö: EUCAST; 2019. Available from: https://www.eucast.org/fileadmin/src/media/PDFs/ EUCAST files/RAST/EUCAST_RAST_Breakpoint_Table_v_1.1 final_PDF.pdf

4. Monecke S, Müller E, Dorneanu OS, Vremeră T, Ehricht R. Molecular typing of MRSA and of clinical Staphylococcus aureus isolates from Iaşi, Romania. PLoS One. 2014;9(5):e97833. https://doi.org/10.1371/journal. pone.0097833 PMID: 24846009

5. Monecke S, Coombs G, Shore AC, Coleman DC, Akpaka P, Borg $M$, et al. A field guide to pandemic, epidemic and sporadic clones of methicillin-resistant Staphylococcus aureus. PLoS One. 2011;6(4):e17936. https://doi.org/10.1371/journal. pone.0017936 PMID: 21494333

6. Earls MR, Coleman DC, Brennan GI, Fleming T, Monecke S, Slickers $\mathrm{P}$, et al. Intra-hospital, inter-hospital and intercontinental spread of ST78 MRSA from two neonatal intensive care unit outbreaks established using whole-genome sequencing. Front Microbiol. 2018;9(1485):1485. https://doi. org/10.3389/fmicb.2018.01485 PMID: 30022976

7. Chen S, Zhou Y, Chen Y, Gu J. fastp: an ultra-fast all-in-one FASTQ preprocessor. Bioinformatics. 2018;34(17):i88490. https://doi.org/10.1093/bioinformatics/bty560 PMID: 30423086

8. Bankevich A, Nurk S, Antipov D, Gurevich AA, Dvorkin M, Kulikov AS, et al. SPAdes: a new genome assembly algorithm and its applications to single-cell sequencing. J Comput Biol. 2012;19(5):455-77. https://doi.org/10.1089/cmb.2012.0021 PMID: 22506599

9. Earls MR, Kinnevey PM, Brennan GI, Lazaris A, Skally M, O'Connell B, et al. The recent emergence in hospitals of multidrug-resistant community-associated sequence type 1 and spa type t127 methicillin-resistant Staphylococcus aureus investigated by whole-genome sequencing: Implications for Screening. PLoS One. 2017;12(4):e0175542. https://doi. org/10.1371/journal.pone.0175542 PMID: 28399151

10. Manara S, Pasolli E, Dolce D, Ravenni N, Campana S, Armanini F, et al. Whole-genome epidemiology, characterisation, and phylogenetic reconstruction of Staphylococcus aureus strains in a paediatric hospital. Genome Med. 2018;10(1):82. https:// doi.org/10.1186/S13073-018-0593-7 PMID: 30424799

11. Scheithauer S, Trepels-Kottek S, Häfner H, Keller D, Ittel T, Wagner N, et al. Healthcare worker-related MRSA cluster in a German neonatology level III ICU: a true European story. Int J Hyg Environ Health. 2014;217(2-3):307-11. https://doi. org/10.1016/j.ijheh.2013.07.006 PMID: 23953255

12. Monecke S, Jatzwauk L, Müller E, Nitschke H, Pfohl K, Slickers $P$, et al. Diversity of SCCmec elements in Staphylococcus aureus as observed in south-eastern Germany. PLoS One. 2016;11(9):e0162654. https://doi.org/10.1371/journal. pone.0162654 PMID: 27648947 
13. Loncaric I, Künzel F, Licka T, Simhofer H, Spergser J, Rosengarten R. Identification and characterization of methicillin-resistant Staphylococcus aureus (MRSA) from Austrian companion animals and horses. Vet Microbiol. 2014;168(2-4):381-7. https://doi.org/10.1016/j. vetmic.2013.11.022 PMID: 24332703

14. Loncaric I, Stalder GL, Mehinagic K, Rosengarten R, Hoelzl $\mathrm{F}$, Knauer F, et al. Comparison of ESBL--and AmpC producing Enterobacteriaceae and methicillin-resistant Staphylococcus aureus (MRSA) isolated from migratory and resident population of rooks (Corvus frugilegus) in Austria. PLoS One. 2013;8(12):e84048. https://doi.org/10.1371/journal. pone.0084048 PMID: 24391878

15. Alba P, Feltrin F, Cordaro G, Porrero MC, Kraushaar B, Argudín $M A$, et al. Livestock-associated methicillin resistant and methicillin susceptible Staphylococcus aureus sequence type (CC) 1 in european farmed animals: high genetic relatedness of isolates from Italian cattle herds and humans. PLoS One. 2015;10(8):e0137143. https://doi.org/10.1371/journal. pone.0137143 PMID: 26322785

16. Baig S, Johannesen TB, Overballe-Petersen S, Larsen J, Larsen AR, Stegger M. Novel SCCmec type XIII (9A) identified in an ST152 methicillin-resistant Staphylococcus aureus. Infect Genet Evol. 2018;61:74-6. https://doi.org/10.1016/j. meegid.2018.03.013 PMID: 29567305

17. Bartels MD, Boye K, Rohde SM, Larsen AR, Torfs H, Bouchy $P$, et al. A common variant of staphylococcal cassette chromosome mec type IVa in isolates from Copenhagen, Denmark, is not detected by the BD GeneOhm methicillin resistant Staphylococcus aureus assay. J Clin Microbiol. 2009;47(5):1524-7. https://doi.org/10.1128/JCM.02153-08 PMID: 19297600

18. Bartels MD, Hansen LH, Boye K, Sørensen SJ, Westh H. An unexpected location of the arginine catabolic mobile element (ACME) in a USA300-related MRSA strain. PLoS One. 2011;6(1):e16193. https://doi.org/10.1371/journal. pone.0016193 PMID: 21283578

19. Hill-Cawthorne GA, Hudson LO, El Ghany MFA, Piepenburg O, Nair M, Dodgson A, et al. Recombinations in staphylococcal cassette chromosome mec elements compromise the molecular detection of methicillin resistance in Staphylococcus aureus. PLoS One. 2014;9(6):e101419. https://doi.org/10.1371/journal. pone.0101419 PMID: 24972080

\section{License, supplementary material and copyright}

This is an open-access article distributed under the terms of the Creative Commons Attribution (CC BY 4.0) Licence. You may share and adapt the material, but must give appropriate credit to the source, provide a link to the licence and indicate if changes were made.

Any supplementary material referenced in the article can be found in the online version.

This article is copyright of the authors or their affiliated institutions, 2020. 